\title{
Performance evaluation of H264/SVC streaming system featuring real-time in-network adaptation
}

\author{
Nikolaos Zotos, Georgios Xilouris \\ Institute of Informatics and Telecommunications \\ NCSR Demokritos \\ Athens, Greece \\ \{nzotos, xilouris\}@iit.demokritos.gr \\ Daniele Renzi \\ bSoft Ltd \\ Macerata, Italy \\ daniele@bsoft.net
}

\author{
Beilu Shao \\ KEYMILE AG \\ Bern, Switzerland \\ beilu.shao@keymile.com
}

\author{
Anastasios Kourtis \\ Institute of Informatics and Telecommunications \\ NCSR Demokritos \\ Athens, Greece \\ kourtis@iit.demokritos.gr
}

\begin{abstract}
In the recent years one of the most active research topics in multimedia networking is the exploitation of the Scalable Video Coding (SVC) as a scalable solution for efficient network resources utilization. SVC introduces scalability by exploiting a layered encoding of the video stream, thus enabling real-time in-network adaptation by selectively allowing the transmission of appropriate layers. This paper presents an architecture that exploits SVC capabilities in order to provide end-to-end QoS assurance via in-network video adaptation. The adaptation system management is based on MPEG-21 framework while the network QoS mechanisms are based on DiffServ standard. The performance evaluation of the proposed architecture is performed over a real test-bed infrastructure.
\end{abstract}

Keywords: in-network adaptation, SVC, QoS, DiffServ, end-to-end, streaming, H264

\section{INTRODUCTION}

With the latest development of technologies, real-time multimedia applications have gained much attention and contribution from both academia and industry. The developed technologies fields span across many stages of the content delivery chain (i.e. video coding, channel coding, networking architectures, QoS techniques, terminal devices). At the same time, a variety of new media content formats, standardcompliant or proprietary, have emerged resulting in a diversity of media itself. In summary, multimedia delivery has been growing from single format video transmission over the monolithic data service network to the adaptive delivery of the complex multimedia across heterogeneous networks, terminals, and users [1].

Particularly, recent advances in video coding have led to the new standard SVC, which enables the scalability in spatial, temporal, and SNR quality, while keeping compression at high efficiency [2]. Such benefits remove the restriction of the conventional rate adaptation architectures, i.e., bit rate adaptation on the media server or the real-time encoder for appropriate channel rate, possibly with feedback supports from the receivers. A more practical and valuable system topology, was firstly introduced in [3] for H.264/MPEG-4 AVC and in [4] for SVC, is the Media Aware Network Element (MANE) which performs in-network adaptation [5]. In this architecture, the terminal can request the adaptation using a feedback signaling that carries QoS metrics, which can be processed by the MANE to enforce adaptation decisions. In this paper, we present performance evaluation of a comprehensive framework for scalable video streaming with in-network adaptation for heterogeneous delivery [5]. The adaptive streaming architecture consists of a server with a real-time SVC encoder, an adaptive network node assisted with an MPEG- 21 adaptation decision taking engine (ADTE) [6], and a terminal with appropriate perceptual quality, network conditions and user preferences for adaptation support. Finally, an experimental network testbed has been configured supporting DiffServ mechanism for the end-to-end QoS provisioning. The paper is organized as follows, Section 2 presents the adaptive video streaming architecture while sections 3, 4 present the laboratory test-bed and the evaluation results. Finally, Section 5 concludes.

\section{ADAPTIVE SCALABLE VIDEO STREAMING SYSTEM}

This section presents the proposed in-network adaptive video streaming architecture based on SVC (Fig. 1). The system is composed of the Server, the Adaptation Node (MANE), and a distributed Terminal, controlled by a service manager, the Integrated Management Supervisor (IMS). The system is designed to make multimedia content management more effective by integrating content and context. After initial system setup with IMS, the Server encodes and streams content and context in real-time to the Adaptation Node.

The Adaptation Node adapts the video based on ADTE's decision from the QoS mapping between scalable content and contextual constraints, imposed by network conditions, 
terminal capabilities, and human user preferences. The decoder of the Terminal Client decodes the adapted video and feeds back the monitored user quality periodically to the IMS via the Terminal Server.

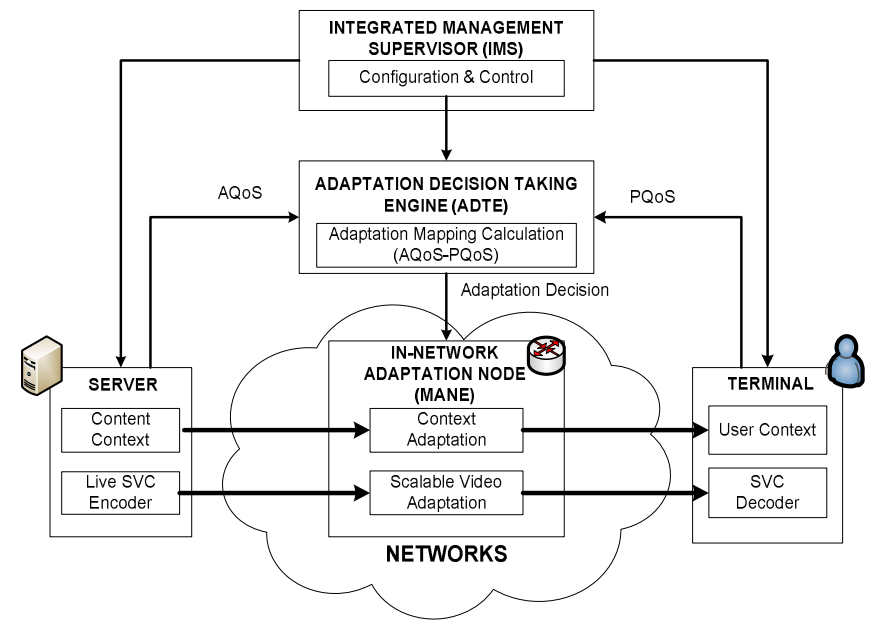

Figure 1. The In-network adaptive SVC streaming architecture

To enhance the inter-operable (semi-) automatic machineto-machine interaction over the network, all these modules are efficiently designed in Web Service.

\section{EVALUATION TESTBED}

\section{A. Testbed architecture configuration}

In order to perform the appropriate performance experiments, a test-bed has been developed that supports the gathering of statistics from all test-bed's building blocks. Measured statistics, collected throughout the experimental sessions are afterwards processed off-line.

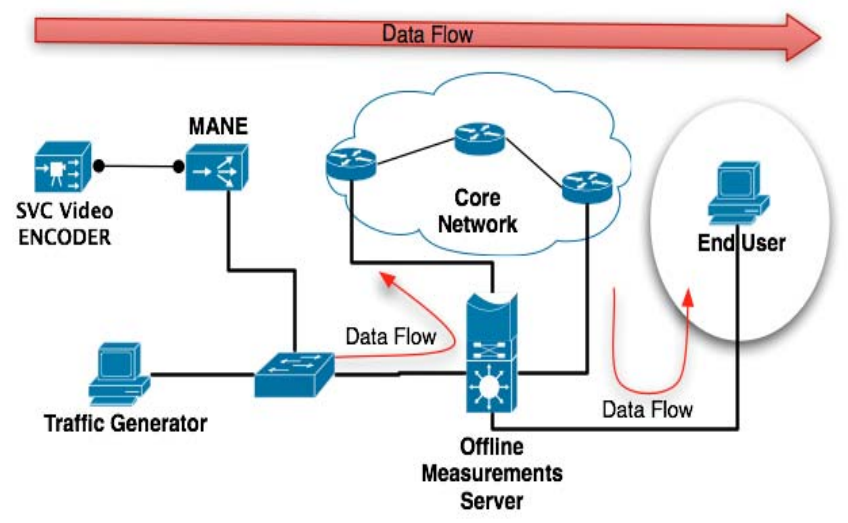

Figure 2. Overall testbed architecture

The proposed test-bed is depicted in Fig. 10. The test-bed is comprised of four parts: (i) the Content Provider part, (ii) the in-network adaptation part, (iii) the Core Network part, and (iv) the end-user part. The Content Provider is realized with a
H264/SVC live streaming encoder, from where the End User requests and receives the content.

The in-network adaptation part is realized through the MANE node. In this node the SVC video stream is adapted, according to the decisions made by the IMS and the forwarded to the initial destination. The traffic always passes through this node even when no adaptation is enforced at the video stream. In this notion the MANE can actually be seen as a common router.

\section{B. Perceived QoS mapping}

The proposed architecture can be modified to accommodate any QoS models and PQoS mapping. The adaptation decisionmaking process is acknowledged about the Perceived QoS to Application QoS mapping to get a correspondence for adaptation. Compared with standard quality measurement model, adaptation based QoS measurement is very different, which considers how to characterize the relationship between the calculated QoS and the adaptation control parameters. Table 1 demonstrates a simple scenario used for the experiments, performing frame rate adaptation by temporal scalability. The frame rate is the main PQoS parameter, and the perceived frame rate corresponds to a certain SVC temporal layer in the source stream.

TABLE I. SCENARIO SETUP OVERVIEW

\begin{tabular}{|c|c|c|c|}
\hline User QoS Preferences & Gold & Silver & Bronze \\
\hline SVC Temporal Layer & 2 & 1 & 0 \\
\hline Frame Rate[fps] & 25.000 & 12.500 & 6.250 \\
\hline Target PQoS [scale 0 - 100] & 85 & 70 & 55 \\
\hline PQoS Interval [scale 0 - 100] & 10 & 10 & 10 \\
\hline PQoS Range [scale 0 - 100] & $80-90$ & $65-75$ & $50-60$ \\
\hline
\end{tabular}

\section{EXPERIMENTAL RESULTS}

In this section, we quantitatively present the results of the aforementioned architecture under various network traffic scenarios. The conducted experiments involve three scenarios based on how the video traffic is classified in terms of BA. In the first scenario, the video traffic is marked and policed as EF at ingress router. In the second scenario, the video traffic is marked and policed as AF11 at the ingress router. In the third scenario, the video traffic is again marked as AF11 but no policing is performed. Each scenario experiment is classified according the total load of traffic and the type of reservation (policing). The video traffic is policed in two different ways, the first one is using the average video rate as an upper limit for the policer and the second is to use the peak video rate. Table 2 presents the overall scenario setup. The test video sequence is a 180 second video clip that is encoded live from the H.264/SVC encoder and streamed to the End User Terminal. The video contains various levels of spatial and temporal complexity. 
TABLE II SCENARIO SETUP OVERVIEW

\begin{tabular}{|c|c|c|c|c|}
\hline Scenario & $\begin{array}{c}\text { Video Flow } \\
\text { Marking }\end{array}$ & Policer & Reservation Rate & $\begin{array}{c}\text { Load } \\
(\%)\end{array}$ \\
\hline \multirow{3}{*}{ EFp } & \multirow{3}{*}{$\mathrm{EF}$} & Yes & Peak (700 kbps) & 100 \\
\hline & & Yes & Average (300 kbps) & 100 \\
\hline & & Yes & Average (300 kbps) & 110 \\
\hline \multirow{3}{*}{ AFp } & \multirow{3}{*}{ AF11 } & Yes & Average (300 kbps) & 100 \\
\hline & & Yes & Average (300 kbps) & 110 \\
\hline & & Yes & Average (300 kbps) & 120 \\
\hline \multirow{2}{*}{$\mathbf{A F}$} & \multirow{2}{*}{ AF11 } & No & Average (300 kbps) & 100 \\
\hline & & No & Average (300 kbps) & 110 \\
\hline
\end{tabular}

The observed peak bit rate of the video is $750 \mathrm{kbps}$ and the average bit rate is around $350 \mathrm{kbps}$. These are the parameters used at the policer in order to produce various test cases. MGEN traffic generator is employed to keep the configured network queues at their upper bandwidth threshold; this traffic is called cross traffic (CT). Figures 3, 4, 5 depict the overall experimental results for the 3 scenarios, including average PQoS percentage, average bitrate and average loss percentage.

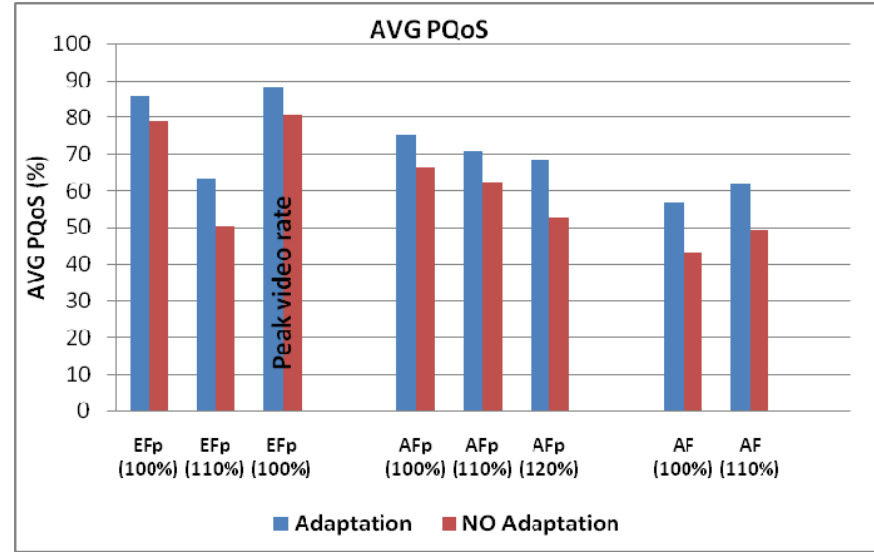

Figure 3. AVG PQoS with and without adaptation

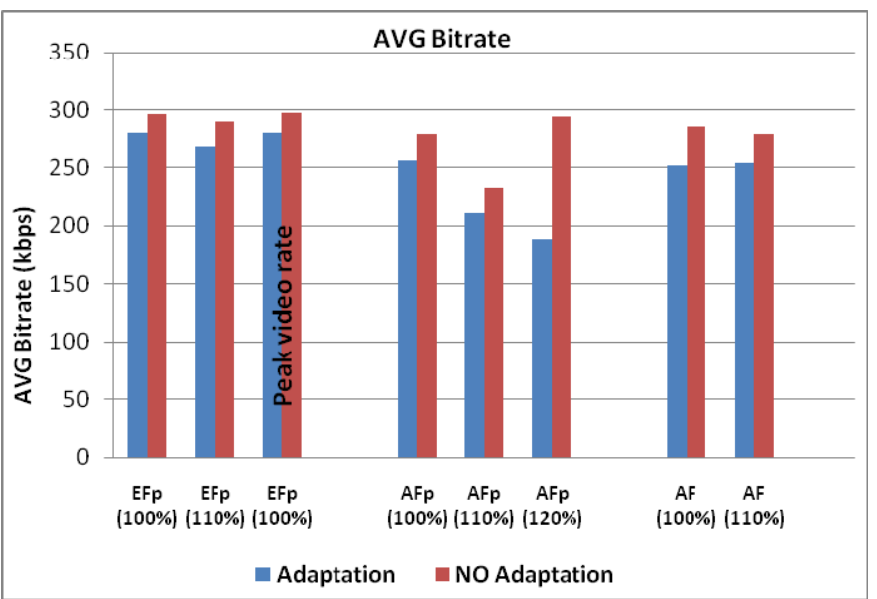

Figure 4. AVG video bitrate with and without adaptation

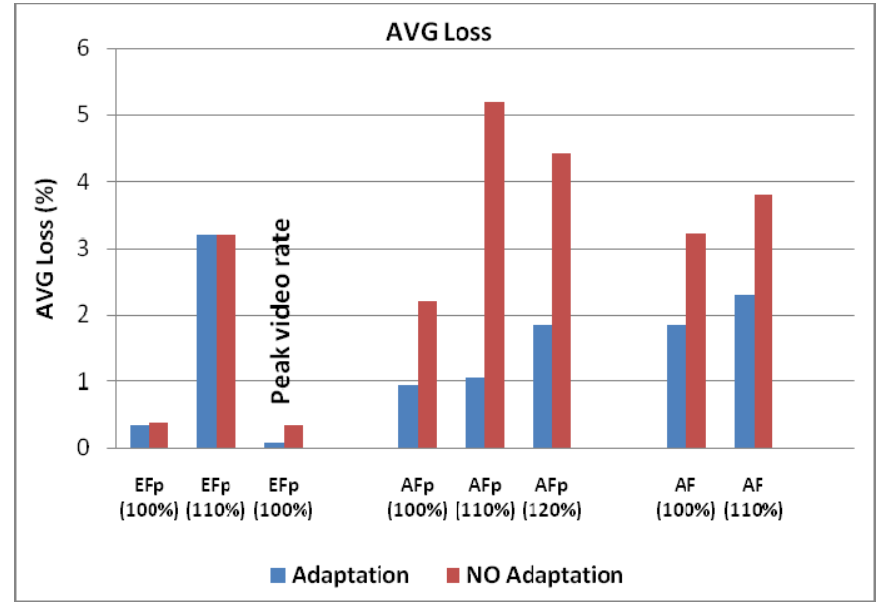

Figure 5. AVG packet loss with and without adaptation

\section{CONCLUSIONS}

This paper presented a real-time video streaming system envisaging in-network media adaptation exploiting a contentaware network node. The video streaming systems introduces a cross layer QoS mapping based framework for media and user/terminal aware transmission and management. Furthermore MPEG-21 and Web Services are utilized to provide universal media access over heterogeneous networks and terminals. The system was evaluated for its performance and efficiency in a laboratory testbed setup featuring a full end-to-end network infrastructure. The results demonstrate the efficiency in the exploitation of the available network resources in relation to the QoE provided to the end user.

\section{ACKNOWLEDGEMENT}

Part of the work presented was funded by the EU and supported by the IST-ENTHRONE (IST-507637) Project. Continuation of this work is conducted in the frame of ICTALICANTE (ICT- 248652) EU funded project.

\section{REFERENCES}

[1] Burnett I., Van de Walle R., Hill K., Bormans J., Pereira F., MPEG-21: goals and achievements, IEEE MultiMedia, 2003, 10(6), pp. 60-70.

[2] Schwarz H., Marpe D., Wiegand T., Overview of the scalable video coding extension of the H.264/AVC standard, IEEE Trans. Circuits Syst. Video Technol., 2007, vol 17, no. 9, pp. 1103-1120.I.

[3] ISO/IEC JTC1/SC29/WG11, Applications and requirements for scalable video coding, Doc. N6880, Jan. 2005.

[4] Schierl T., Stockhammer T., Wiegand T., Mobile video transmission using scalable video coding, IEEE Trans. Circuits Syst. Video Technol., 2007, vol 17, no. 9, pp. 1204-1217.

[5] R. Kuschnig, I. Kofler, M. Ransburg, H. Hellwagner, "Design options and comparison of in-network H.264/SVC adaptation", Journal of Visual Communication and Image Representation, Sept. 2008.

[6] I. Kofler, J. Seidl, C. Timmerer, H. Hellwagner, I. Djama and T. Ahmed, "Using MPEG-21 for cross-layer multimedia content adaptation", Journal on Signal, Image and Video Processing, Springer, vol. 2, no. 4, Dec. 2008. 